\title{
Disseminated Cryptococcosis in a 63-year-old Patient with Multiple Sclerosis Treated with Fingolimod
}

\author{
Hiroyuki Seto ${ }^{1}$, Mitsushige Nishimura ${ }^{1}$, Katsuhiro Minamiji ${ }^{1}$, Sonoko Miyoshi ${ }^{1}$, \\ Hiroyuki Mori ${ }^{1}$, Kenji Kanazawa ${ }^{1}$ and Hisafumi Yasuda ${ }^{1,2}$
}

\begin{abstract}
We herein report the case of a 63-year-old man who presented with a 3-month history of a cutaneous nodular lesion of his jaw, low grade fever, lethargy and progressive cognitive impairment. He had a 30-year history of multiple sclerosis and had been treated with fingolimod for the previous 2 years. Laboratory data revealed CD4 lymphocytopenia and a tissue culture of the skin nodule was positive for Cryptococcus neoformans. Cerebrospinal fluid and serum cryptococcal antigen tests were also positive and we diagnosed him to have disseminated cryptococcosis. This dissemination might be associated with fingolimod-induced CD4 lymphocytopenia. The risk of an opportunistic infection should therefore be considered when encountering fingolimod-treated patients.
\end{abstract}

Key words: cryptococcosis, multiple sclerosis, fingolimod, CD4 lymphocytopenia

(Intern Med 55: 3383-3386, 2016)

(DOI: 10.2169/internalmedicine.55.7255)

\section{Introduction}

Cryptococcosis is an opportunistic fungal infection caused by Cryptococcus species, and Cryptococcus neoformans is considered to be a major primary pathogen. Although exposure to Cryptococcus species is common, the development of symptomatic disease usually requires immunosuppression. With an increasing number of immunosuppressed patients because of human immunodeficiency virus (HIV) infection, solid organ transplantation, and the clinical use of potent immunosuppressive agents, such as cancer chemotherapy, monoclonal antibodies, and corticosteroids, cryptococcosis has now become a common infectious complication (1).

Fingolimod is an oral Food and Drug Administrationapproved medication for adults with relapsing-remitting multiple sclerosis (MS). Fingolimod is a sphingosine 1phosphate (S1P) receptor modulator which prevents the egress of lymphocytes from the lymph nodes (2). Because of its mechanism of action, the risk of opportunistic infectious complication, such as varicella zoster virus infection, has been reported $(3,4)$. Recently, three case reports of
Cryptococcus infection associated with fingolimod have been documented (5-7).

\section{Case Report}

A 63-year-old man with a history of MS was referred to our hospital for a detailed investigation of an erythematous nodular lesion with ulceration present on his left mandible. Three months prior to referral, he developed a papular lesion on his left lower jaw, low-grade fever, lethargy, and muscle weakness. The cutaneous lesion had gradually increased in size and eventually developed into an ulcer. One month prior to this referral, lower-extremity muscle weakness and cognitive impairment worsened and he could not read or write correctly. He was referred to our dermatology department for an investigation of the cutaneous lesion.

The case had a 30-year history of MS with poor responses to disease-modifying therapy, and two years prior to referral, treatment with fingolimod had been started. Nifedipine, valsartan, clopidogrel, aspirin, and atorvastatin had also been administered for hypertension and coronary heart disease in his past medical history. Since the start of

${ }^{1}$ Department of General Internal Medicine, Kobe University Hospital, Japan and ${ }^{2}$ Division of Health Sciences, Department of Community Health Sciences, Kobe University Graduate School of Health Sciences, Japan

Received for publication February 9, 2016; Accepted for publication March 16, 2016

Correspondence to Dr. Hiroyuki Seto, seto-kob@umin.ac.jp 


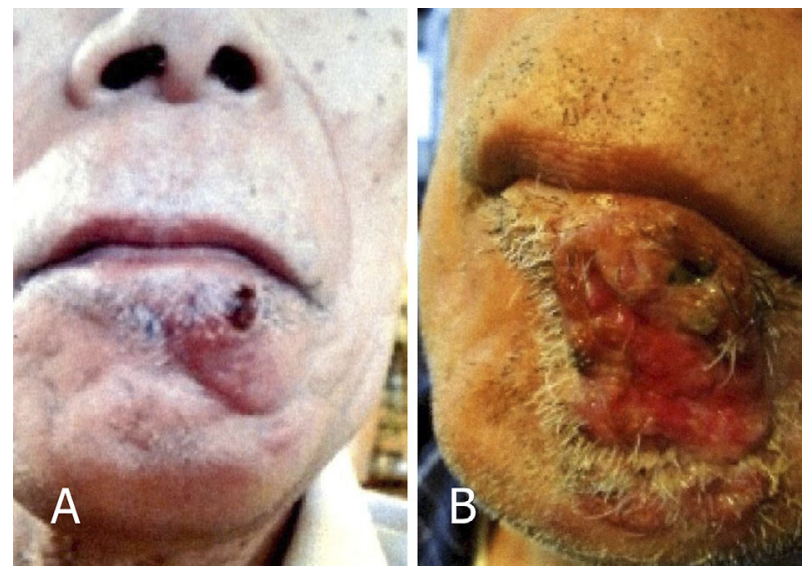

Figure 1. A cutaneous lesion of the left mandible. A: Three months prior to admission. There was an erythematous nodule beneath the lower lip. B: On admission, there was an erythematous multilocular lesion with ulcer that measured $5 \times 3 \mathrm{~cm}$ in size on his left mandible.

fingolimod treatment, his lymphocyte counts were approximately $500 / \mu \mathrm{L}$. He denied any recent travel or close contact with birds.

On examination, he was not in acute distress, but his speech was slow and his accent was flat. He had no fever. He had a normal blood pressure $(122 / 64 \mathrm{mmHg}$ ) and heart rate $(60 \mathrm{bpm})$. His respiratory rate and oxygen saturation were 16 per minute and $99 \%$ in room air, respectively. There was an erythematous multilocular lesion with ulceration that measured $5 \times 3 \mathrm{~cm}$ on his left mandible (Fig. 1). There was slight neck stiffness. Cardiovascular, pulmonary, and abdominal examinations were normal. A neurological examination revealed left lateral gaze palsy, left central facial paralysis, slight dysarthria, and limb-kinetic apraxia. His muscle strength and tendon reflex of the upper extremities were normal and his bilateral lower-extremity muscle strength was $2 / 5$ (an inability to overcome gravity). Positive findings for Babinski's reflex and foot clonus were seen bilaterally. These neurological findings, except for lowerextremity muscle weakness, were the same as they had been before referral.

To investigate the cause of the skin lesion, a skin biopsy was performed. The histopathology of the skin biopsy revealed no malignant cells. However, many yeast-like organisms with thick capsules were observed in the skin specimens and periodic acid-Schiff and Grocott staining were positive for these organisms (Fig. 2). C. neoformans was isolated and identified from a tissue culture. Both a tissue culture and polymerase chain reaction for Mycobacterium tuberculosis were negative.

Computed tomography (CT) of the chest revealed multinodular lesions in the left lung. Both staining and a culture of his sputum showed no pathogen growth. Bronchoscopy for lung nodular lesions was not performed. Fluid attenuated inversion recovery images of brain magnetic resonance imaging (MRI) showed multiple hyperintense oval lesions in the white matter. The same oval lesions observed by brain MRI performed one year prior to this episode were present. Therefore, these lesions were most likely due to MS.

A cerebrospinal fluid (CSF) examination yielded elevated white blood cell numbers $(74 / \mu \mathrm{L})$ and protein $(323 \mathrm{mg} / \mathrm{dL})$, and the titer of cryptococcal antigen by latex agglutination of CSF was 1:1,024. The opening pressure of a lumbar puncture was $16 \mathrm{~cm} \mathrm{H}_{2} \mathrm{O}$. Mononuclear and polynuclear white cells in the CSF were present at $90 \%$ and $10 \%$ of overall white blood cells, respectively. However, gram staining, India ink staining and a culture of CSF showed no bacterial or fungal pathogens.

Repeated blood cultures were all negative for bacterial and fungal pathogens. Laboratory investigations, including liver enzyme levels, renal function and electrolytes, were all within the normal limits, but the titer of serum cryptococcal antigen was $1: 256$. White blood cell counts were $5,000 / \mu \mathrm{L}$ (neutrophils $82 \%$, lymphocytes 6\%, monocytes 8\%). Absolute lymphocyte counts were $300 / \mu \mathrm{L}\left(\mathrm{CD}^{+}\right.$lymphocytes $145 / \mu \mathrm{L}, \mathrm{CD}^{+}$lymphocytes $\left.113 / \mu \mathrm{L}\right)$. Antigen and antibody tests for HIV were negative.

We reached a diagnosis of disseminated cryptococcal infection based on a tissue culture of the skin biopsy, and positive findings for CSF and the serum cryptococcal antigen test. After admission, the administration of fingolimod was stopped because of lymphocytopenia, which might have led to an immunocompromised state. Liposomal amphotericin B $300 \mathrm{mg} /$ day ( $5 \mathrm{mg} / \mathrm{kg} /$ day) and flucytosine 4,000 $\mathrm{mg} /$ day $(64 \mathrm{mg} / \mathrm{kg} /$ day) were administered for 6 weeks as induction therapy. Thereafter, consolidation therapy for 8 weeks with fluconazole $400 \mathrm{mg}$ orally daily was administered.

Two weeks after the initiation of induction therapy, we repeated a CSF examination and observed a decrease in the number of white blood cells $(49 / \mu \mathrm{L})$ and protein $(177 \mathrm{mg} /$ dL). After 4 weeks of induction therapy, the absolute lymphocyte counts returned to normal levels $(1,239 / \mu \mathrm{L})$ and a follow-up study with chest CT showed diminished nodular lesions in the left lung. After 6 weeks of induction therapy, his cutaneous lesion had almost completely healed. However, the lower-extremity muscle weakness and cognitive impairment had only slightly improved, though no obvious exacerbation of MS was seen. He was transferred to a regional hospital for continuous care and rehabilitation.

\section{Discussion}

Fingolimod is a S1P receptor modulator with an immunomodulatory and disease-modifying activity in MS patients (2). A previous study reported that fingolimod reduced the frequency of clinical exacerbations and delayed the accumulation of physical disability in adults with relapsing forms of MS (8). The S1P receptor regulates lymphocyte egress from both the thymus and peripheral lymphoid organs and is essential for lymphocyte recirculation. Therefore, the mechanism of action of fingolimod-phosphate is to 


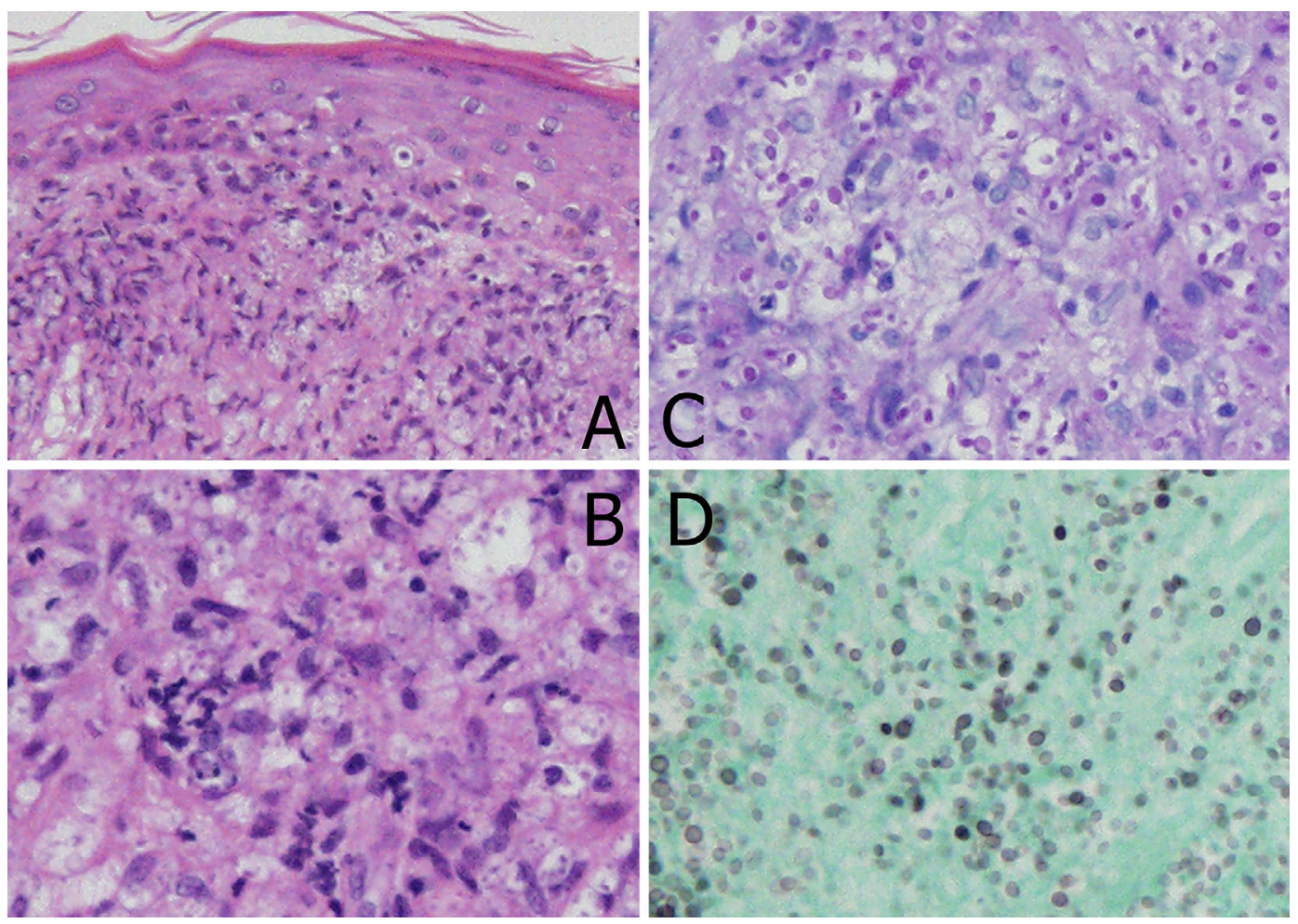

Figure 2. Light microscopy findings of a skin biopsy specimen from the left mandible. A: The biopsy specimen revealed diffuse inflammatory infiltration [magnification $\times 100$, Hematoxylin and Eo$\sin ($ H\&E) staining]. B: Many yeast cells with a thick capsule (magnification $\times 200$, H\&E staining). C: Numerous periodic acid-Schiff (PAS) -positive encapsulated yeast cells (magnification $\times 200$, PAS). D: Grocott staining of the biopsy specimen showed numerous yeast cells that were black in color (magnification $\times 200$, Grocott).

inhibit the S1P receptor function, thus preventing lymphocyte egress from the lymph nodes, reducing the number of lymphocytes in the peripheral blood and ultimately preventing them from entering the central nervous system (CNS) (9, 10). Fingolimod induced a dose-dependent reduction in the peripheral lymphocyte counts to $20-30 \%$ of the baseline value (2), but it also increases the risk of infections, such as varicella zoster virus $(3,4)$. Although fingolimod has been reported to reduce the number of both $\mathrm{CD}^{+}$and $\mathrm{CD}^{+} \mathrm{T}$ cells, the effect was more pronounced for the $\mathrm{CD} 4^{+}$ T-cell subset (11).

C. neoformans is a basidiomycetous encapsulated yeast that is ubiquitous in the environment. Cryptococcosis is an invasive fungal infection caused by $C$. neoformans or $C$. gattii. Most patients with symptomatic disseminated cryptococcosis have an underlying immunocompromised condition, such as HIV infection, prolonged treatment with corticosteroids, organ transplantation, advanced malignancy, diabetes, or sarcoidosis $(1,12)$. The development of cryptococcosis is also associated with idiopathic CD4 lymphocytopenia (13) or the use of specific immune-modifying agents, such as alemtuzumab, infliximab, etanercept, or adalimumab $(14,15)$.

To the best of our knowledge, only three case reports of cryptococcal infection associated with fingolimod treatment have been reported to date (5-7). Including the present case, the CD4 cell counts were obtained for 3 of the 4 cases, and these 3 cases all demonstrated CD4 lymphocytopenia. Although a causal link between fingolimod-induced CD4 lymphocytopenia and cryptococcosis was not proven directly, the decreased CD4 cell counts associated with fingolimod might be a risk factor for acute cryptococcal infection or a reactivation of a latent infection. The administration of fingolimod should be immediately stopped in patients who develop a serious infection and it should not be initiated again until the infection has completely resolved. Further investigations are needed to clarify the relationship between fingolimod treatment and cryptococcal infection, and elucidate whether fingolimod can be reintroduced safely in patients with cryptococcosis.

C. neoformans has been reported to infect any organ, while the two most common sites for infection are the lung and CNS, followed by skin. A common skin lesion is the papule or maculopapule with a soft or ulcerated center. Some lesions in severely immunosuppressed patients resemble molluscum contagiosum, and they can mimic squamous carcinoma or basal cell carcinoma (16). Because of the variety of associated skin manifestations, a correct diagnosis requires a biopsy with proper histopathology and culture analyses. In most cases, these skin lesions represent disseminated infection. For the diagnosis of a cryptococcal infection, suspicious skin lesions should be biopsied, and if a 
cryptococcal skin infection is diagnosed, then proactive screening of disseminated lesions, especially in the CNS is necessary.

The mortality associated with cryptococcosis in HIVnegative patients is approximately $30 \%$ (17-19). Unrecognized and untreated cryptococcosis can thus be fatal, especially in immunocompromised patients, and untreated cryptococcal meningoencephalitis is uniformly fatal. Although our patient survived and the cutaneous lesion healed successfully, the impaired activity of daily life and reduced cognitive function persisted. Early recognition and appropriate treatment is therefore important to reduce morbidity and prevent progression to a potentially life-threatening CNS disease.

Cryptococcal infection should be considered in MS patients treated with fingolimod who have subacute symptoms, such as skin lesions, low grade fever, lethargy or cognitive impairment, especially if lymphocytopenia or decreased CD4 cell counts are observed. As in the case of HIV infection or idiopathic CD4 lymphocytopenia, regular monitoring of CD4 cell counts is thus considered to be warranted in patients treated with fingolimod. Neurologists and general practitioners should therefore be aware of the potential risk of cryptococcal infections, and possibly other opportunistic infections in patients treated with fingolimod.

The authors state that they have no Conflict of Interest (COI).

\section{Acknowledgement}

The authors would like to sincerely thank Dr. Yasuko Hirano, Dr. Takehiro Toyokuni and Dr. Takashi Arai for their valuable contributions to the patient care. The authors are also deeply grateful to Dr. Shungo Yamamoto for insightful comments and suggestions on draft of this manuscript.

\section{References}

1. Chayakulkeeree M, Perfect JR. Cryptococcosis. Infect Dis Clin North Am 20: 507-544, v-vi, 2006.

2. Kappos L, Antel J, Comi G, et al. Oral fingolimod (FTY720) for relapsing multiple sclerosis. N Engl J Med 355: 1124-1140, 2006.

3. Ratchford JN, Costello K, Reich DS, Calabresi PA. Varicellazoster virus encephalitis and vasculopathy in a patient treated with fingolimod. Neurology 79: 2002-2004, 2012.

4. Arvin AM, Wolinsky JS, Kappos L, et al. Varicella-zoster virus in- fections in patients treated with fingolimod: risk assessment and consensus recommendations for management. JAMA Neurol 72: 31-39, 2015.

5. Huang D. Disseminated cryptococcosis in a patient with multiple sclerosis treated with fingolimod. Neurology 85: 1001-1003, 2015.

6. Achtnichts L, Obreja O, Conen A, Fux CA, Nedeltchev K. Cryptococcal meningoencephalitis in a patient with multiple sclerosis treated with fingolimod. JAMA Neurol 72: 1203-1205, 2015.

7. Forrestel AK, Modi BG, Longworth S, Wilck MB, Micheletti RG. Primary cutaneous cryptococcus in a patient with multiple sclerosis treated with fingolimod. JAMA Neurol 73: 355-356, 2016.

8. Kappos L, Radue EW, O'Connor P, et al. A placebo-controlled trial of oral fingolimod in relapsing multiple sclerosis. N Engl $\mathrm{J}$ Med 362: 387-401, 2010.

9. Matloubian M, Lo CG, Cinamon G, et al. Lymphocyte egress from thymus and peripheral lymphoid organs is dependent on S1P receptor 1. Nature 427: 355-360, 2004.

10. Brinkmann V, Cyster JG, Hla T. FTY720: sphingosine 1-phosphate receptor-1 in the control of lymphocyte egress and endothelial barrier function. Am J Transplant 4: 1019-1025, 2004.

11. Chun J, Hartung HP. Mechanism of action of oral fingolimod (FTY720) in multiple sclerosis. Clin Neuropharmacol 33: 91-101, 2010.

12. Bernard C, Maucort-Boulch D, Varron L, et al. Cryptococcosis in sarcoidosis: cryptOsarc, a comparative study of 18 cases. QJM 106: 523-539, 2013.

13. Zonios DI, Falloon J, Huang CY, Chaitt D, Bennett JE. Cryptococcosis and idiopathic CD4 lymphocytopenia. Medicine (Baltimore) 86: 78-92, 2007.

14. Nath DS, Kandaswamy R, Gruessner R, Sutherland DE, Dunn DL, Humar A. Fungal infections in transplant recipients receiving alemtuzumab. Transplant Proc 37: 934-936, 2005.

15. Tsiodras S, Samonis G, Boumpas DT, Kontoyiannis DP. Fungal infections complicating tumor necrosis factor alpha blockade therapy. Mayo Clin Proc 83: 181-194, 2008.

16. Negroni R. Cryptococcosis. Clin Dermatol 30: 599-609, 2012.

17. Kiertiburanakul S, Wirojtananugoon S, Pracharktam R, Sungkanuparph S. Cryptococcosis in human immunodeficiency virus-negative patients. Int J Infect Dis 10: 72-77, 2006.

18. Lee YC, Wang JT, Sun HY, Chen YC. Comparisons of clinical features and mortality of cryptococcal meningitis between patients with and without human immunodeficiency virus infection. J Microbiol Immunol Infect 44: 338-345, 2011.

19. Pappas PG, Perfect JR, Cloud GA, et al. Cryptococcosis in human immunodeficiency virus-negative patients in the era of effective azole therapy. Clin Infect Dis 33: 690-699, 2001.

The Internal Medicine is an Open Access article distributed under the Creative Commons Attribution-NonCommercial-NoDerivatives 4.0 International License. To view the details of this license, please visit (https://creativecommons.org/licenses/ by-nc-nd/4.0/).

(C) 2016 The Japanese Society of Internal Medicine http://www.naika.or.jp/imonline/index.html 